
c) 2004 Kluwer Academic Publishers. Manufactured in The Netherlands.

\title{
Cycle Action on Treelike Structures
}

\author{
DANIELE A. GEWURZ \\ gewurz@mat.uniroma1.it \\ FRANCESCA MEROLA \\ Merola@mat.uniroma1.it \\ Dipartimento di Matematica, Università di Roma "La Sapienza", Piazzale Aldo Moro, 2, 00185 Roma, Italy
}

Received January 28, 2003; Revised October 6, 2003; Accepted October 15, 2003

\begin{abstract}
The purpose of this paper is to study the action on cycles of several known classes of oligomorphic groups, that is, infinite permutation groups of countable degree having only finitely many orbits on $k$-sets for each $k$. The groups studied here are all related to trees and treelike relational structures. The sequence whose $k$-th term is the number of orbits in the action on $k$-cycles is called Parker sequence. It turns out that, if we are dealing with the automorphism group of a suitable relational structure, this sequence counts also the finite substructures admitting a cyclic automorphism; in calculating these sequences for various groups, we shall thus describe and enumerate such substructures.
\end{abstract}

Di più dirò: ch'a gli alberi dà vita spirito uman che sente e che ragiona. Per prova sollo; io n'ho la voce udita che nel cor flebilmente anco mi suona.
[I shall say more: the trees are given life by a human spirit that perceives and reasons. I know it by experience: I heard their voice and it still resounds faintly in my heart.]

Torquato Tasso, Gerusalemme liberata, XIII, 49

Keywords: oligomorphic permutation groups, action on cycles, circulant relational structures, trees

\section{Introduction}

In this paper we study treelike structures by calculating the Parker sequences of the automorphism group of a Fraïssé limit of the relevant class of relational structures. Such an automorphism group is oligomorphic: that is, the number of orbits on $k$-subsets of the infinite set it acts on is finite for each $k$. For details see [2]. It turns out that computing the Parker sequence is tantamount to enumerating the circulant structures in the class.

Recall that the Parker sequence (or Parker vector) of a group $G$ is the sequence whose $k$-th term is the number of orbits of $G$ on the set of $k$-cycles appearing in its elements. Parker vectors were first defined for finite groups, in the aim to reconstruct a group from partial information on its structure and action (originally in the context of computational Galois theory). Oligomorphic permutation groups are relevant in this setting because they form a class of infinite groups to which the concept of Parker vector can be extended (see [5]).

The oligomorphic groups we are interested in here are connected with counting trees, sometimes with extra structure, with respect to the number of leaves. Such "treelike structures" and related groups appear in different areas of combinatorics and permutation group theory; we shall follow the approach and notation of Cameron's paper [1]. 
In particular, treelike structures are interesting in the theory of oligomorphic groups because their groups are extremal amongst primitive groups with respect to the growth rate both of their U- and of their L-sequences (see [8] and [9]). For instance, a theorem of Macpherson [8] states that the U-sequence of a primitive permutation group is either the all-1 sequence or grows at least exponentially. Now, most primitive groups show a faster growth rate; the only known examples of oligomorphic groups with exactly exponential growth rate are related to these treelike structures (Sections 2.2 and 2.3) and to local orders (Section 2.1).

\section{Parker sequences for treelike structures}

It is well known (see [2]) that an oligomorphic group $G$ acting on a set $\Omega$ gives rise naturally to two sequences of positive integers: the first one is the L-sequence (from "labelled") $\left(F_{k}\right)$, counting the orbits on $k$-tuples of distinct elements of $\Omega$; the second one is the U-sequence (from "unlabelled") $\left(f_{k}\right)$, which counts the orbits on $k$-subsets of $\Omega$.

There is an important and fruitful connection between combinatorial enumeration and the theory of oligomorphic groups. In what follows, we shall need the concept of Fraïssé class, a class of relational structures with certain suitable properties (see [2]); in particular, the definition embraces such structures as graphs, trees, posets and so on. A Fraïssé class admits a limit, that is a universal countable structure for that class.

If a group is the automorphism group of the limit of a Fraïssé class, it turns out that the Land U-sequences count, respectively, the labelled structures on $k$ points, and the unlabelled ones.

The Parker sequence $\left(p_{k}(G)\right)$ of an oligomorphic group $G$ affords an interpretation in the same vein. The $k$-th component of the Parker sequence, that is the number of orbits of $G$ on the set of $k$-cycles appearing in the elements of $G$, counts the circulant structures in the Fraïssé class on the set $\{0,1, \ldots, k-1\}$ admitting as an automorphism the permutation $(01 \ldots k-1)$ (see [5] for details). This is the point of view we shall adopt in what follows: we shall calculate Parker sequences mostly by counting circulant structures.

\subsection{Local orders}

In this section we are going to study the Fraïssé class of local orders (see [2]; also known as vortex-free tournaments; see [7]) and to show that the entries with odd index $k$ of its Parker sequence are equal to $\varphi(k)$, while the entries with even index are zero. In other words, these are the numbers of circulant local orders on $k$ vertices.

Recall that a tournament is a directed complete graph. We say that a vertex $x$ dominates a vertex $y$ if there is an oriented edge $x \rightarrow y$ from $x$ to $y$. The set of all vertices dominated by $x$ is denoted by $x^{+}$; analogously, we denote by $x^{-}$the set of vertices dominating $x$.

A tournament is said to be a local order if for each 3-cycle $x_{1} \rightarrow x_{2} \rightarrow x_{3} \rightarrow x_{1}$ there is no fourth vertex $y$ dominating or being dominated by all the vertices in the cycle; such a configuration is sometimes called a vortex (see figure 1). So for each vertex $y$, the set $y^{+}$ of all vertices dominated by $y$ contains no 3-cycles and is therefore a total order; the same is true for the set $y^{-}$of all vertices dominating $y$. 

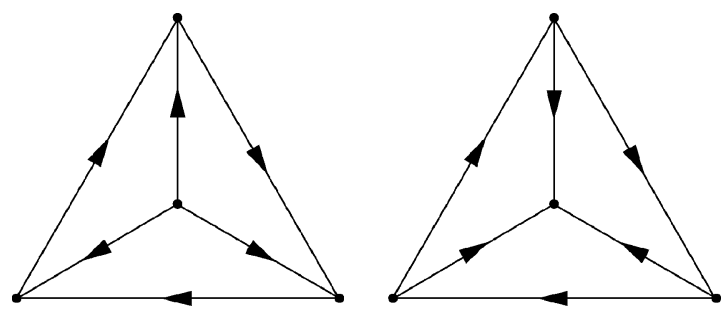

Figure 1. Vortices.

Local orders form a Fraïssé class, the limit of which we denote by $L$. We can describe $L$ by taking as its support a dense subset of the complex roots of unity obtained by choosing exactly one out of each pair $\{\omega,-\omega\}$, and orienting the edges (realised as straight line segments) in such a way that, going along each of them according to its orientation, the center of the unit circle is on the right.

First of all, observe that circulant tournaments, and so in particular local orders, on an even number of vertices cannot exist: circulant local orders must be regular, in the sense that the for each vertex the edges going out are as many as those going in, and this number is $(k-1) / 2$, where $k$ is the number of vertices.

Theorem 2.1 All circulant local orders on the set $\{0,1, \ldots, k-1\}$ admitting the auto-

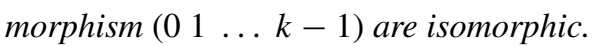

Proof: A particular circulant local order $L_{k}$ on that set affording the automorphism $(01 \ldots k-1)$ is obtained by putting as $i^{+}$the set $\{i+1, \ldots, i+(k-1) / 2\}$ (see figure 2 ). Here and in what follows all calculations are modulo $k$.

Take any circulant local order admitting the cyclic automorphism $\left(\begin{array}{llllll}0 & 1 & 2 & \ldots & k-1\end{array}\right)$ : let us show that it is isomorphic to $L_{k}$. The bijection can be thus described: there is a vertex receiving edges from 0 and from all the vertices dominating 0 but one; call it $a_{1}$. Such a vertex exists because the set $0^{+}$is totally ordered and therefore it possesses a minimum;

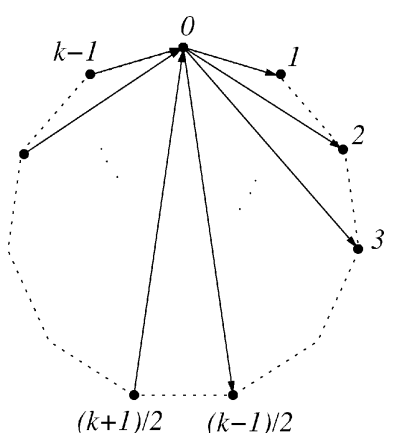

Figure 2. $L_{k}$. 
it is the vertex we are looking for because it dominates all the other vertices in $0^{+}$and therefore, by regularity, is dominated by all the vertices in $0^{-}$but one. Next, there is a vertex $a_{2}$ in the same situation with respect to $a_{1}$, and so on up to $a_{k-1}$. Then the map giving the isomorphism maps 0 to 0 and $i$ to $a_{i}(i>0)$, because for each vertex $a_{i}$ the set $\left\{a_{i+1}, \ldots, a_{i+(k-1) / 2}\right\}$ coincides with $a_{i}^{+}$.

We can say more: $a_{i}$ has to be equal to $i a_{1}$, by circularity. In fact, if $0 \rightarrow a_{1}$ then $b \rightarrow b+a_{1}$, for each vertex $b$, so that $a_{1} \rightarrow 2 a_{1}$ and so on, because by hypothesis the structure admits the automorphism $\left(\begin{array}{llll}0 & 1 & 2 & \ldots\end{array} . k-1\right)$.

So the structure of a circulant local order is determined by the "first neighbour" of 0 , and we get at most $k-1$ different structures. Moreover, if $\left(a_{1}, k\right) \neq 1$, then $\left(1,1+a_{1}, 1+2 a_{1}, \ldots\right)$ is a closed path of the local order; its length $l$ is lesser than $k$ (indeed, $3 \leq l \leq k / 3$ ), so it is dominated by 0 . It is easy to see that the presence of a dominated cycle of length greater or equal than 3 entails the existence of a dominated cycle of length 3 . So in that case the tournament is not vortex-free.

On the other hand, for each $k$ such that $\left(a_{1}, k\right)=1$, the elements $a_{1}, a_{2}=2 a_{1}, \ldots$, $\frac{k-1}{2} a_{1}=a_{\frac{k-1}{2}}$ are all distinct modulo $k$ and thus, reasoning as in the proof of the theorem, the set $\left\{a_{1}, \ldots, a_{(k-1) / 2}\right\}$ can be taken as $0^{+}$. Different values of $a_{1}$ yield different sets.

This proves the following result.

Corollary 2.2 The kth component of the Parker sequence for $\operatorname{Aut}(L)$ is 0 if $k$ is even, and $\varphi(k)$ if $k$ is odd.

\subsection{Trees}

The requirement that a tree admits an automorphism permuting cyclically its leaves is very restrictive and imposes strong limitations to its possible structure.

The structures we are interested in in this (and the next) section were described by Peter Cameron in [1]. In general, we consider a structure given on a set by a quaternary relation induced by a tree $\Delta$ whose leaves (vertices of valency 1 ) are the elements of the set. For the 4-tuple $(a, b, c, d)$ the relation (written as $a b \mid c d$ ) holds if and only if there are in $\Delta$ non-intersecting paths from $a$ to $b$ and from $c$ to $d$. Such relational structures form a Fraïssé class $T$, whose limit is also denoted by $T$ (note that from now on we shall use the same symbol to denote denote a class of structures and its limit).

Recall that a tree is called homeomorphically irreducible (HI) if it has no vertices of valency two. It can be proved [1] that the quaternary relation on the leaves identifies uniquely the underlying HI tree structure (including the structure on internal, non-leaves, nodes). Thus, in what follows, we shall limit ourselves to considering trees as structures defined by the standard binary adjacency relation; the action, however, is on the set of leaves.

We obtain new, related structures by imposing bounds on the valency of the internal vertices. We define the class $T_{n}$ of trees of valency at most $n$, with $n \geq 3$. For $n=3$ we get the so-called boron trees (from the fact that the chemical element boron has valency 3 ), in which all vertices have valency either 1 or 3; see [2] for more. 
We claim that the $k$-th entry of the Parker sequence of $\operatorname{Aut}(T)$ is the number $a_{k}$ of ordered factorisations of $k$, that is the number of ways to express $k$ as a product of integers different from 1, taking two such expressions as distinct if they differ by the ordering of the factors. So $a_{12}=8$, as $12=2 \cdot 6=6 \cdot 2=3 \cdot 4=4 \cdot 3=2 \cdot 2 \cdot 3=2 \cdot 3 \cdot 2=3 \cdot 2 \cdot 2$. For this sequence the following recursion hold:

$$
a_{1}=1, a_{k}=\sum_{d \mid k, d \neq k} a_{d}
$$

So, for instance, $a_{p^{m}}=2^{m-1}$ for $p$ a prime. This is the sequence A002033 in the "Encyclopedia of Integer Sequences" [10].

We shall show that the number of isomorphism types of circulant HI trees with $k$ leaves is equal to the number of ordered factorisations of $k$, and exhibit a bijection between trees and factorisation.

The following result describes the structure of an unlabelled circulant tree in $T$.

Theorem 2.3 The HI tree $\Delta$ is circulant if and only if either $\Delta$ has exactly one or two vertices, or $\hat{\Delta}$, the subtree induced on non leaves, is again a circulant HI tree and in $\Delta$ each leaf of $\hat{\Delta}$ has the same number of children.

Less formally, $\Delta$ has at its centre a single vertex or two joined vertices (sometimes called bicentre), from each of which the same number of edges go out. In all the subsequent "generations" each vertex has the same number of children.

In particular, the stars (trees with just a $k$-valent vertex and $k$ leaves) are circulant trees.

Proof: First note that in $\Delta$ each neighbour of a leaf has the same number of children, since the cyclic automorphism maps leaves to leaves, and consequently it maps neighbours of leaves to neighbours of leaves.

Then observe that if all the leaves of $\Delta$ are deleted, each neighbour of a leaf becomes a leaf; indeed, at least one of the neighbours of a leaf becomes a leaf (because $\Delta$ is a tree, and so also $\hat{\Delta}$, the subtree induced on non-leaves), so they all do.

Now, if $\alpha \in \operatorname{Aut}(\Delta)$ is an automorphism inducing a cyclic permutation on the set leaves of $\Delta$, it induces a permutation $\sigma$ on the set of neighbours of leaves, that is the set of leaves of $\hat{\Delta}$. We claim that $\sigma$ is cyclic. In fact, if $\sigma$ has more than one orbit, then $\alpha$ induces a permutation with at least as many orbits also on the set of leaves of $\Delta$.

We may conclude that the subtree of $\Delta$ induced on non-leaves, $\hat{\Delta}$, is again a circulant tree.

Corollary 2.4 For each $k$, to any ordered factorisation of $k$ corresponds a circulant HI tree on $k$ leaves and vice versa.

Proof: In each "generation" of a circulant HI tree the valency is constant. So the number of its leaves is equal to the product of the numbers of sons in the successive generations, that is either to the valency of the centre (if any) times the product of one less than the valencies of 


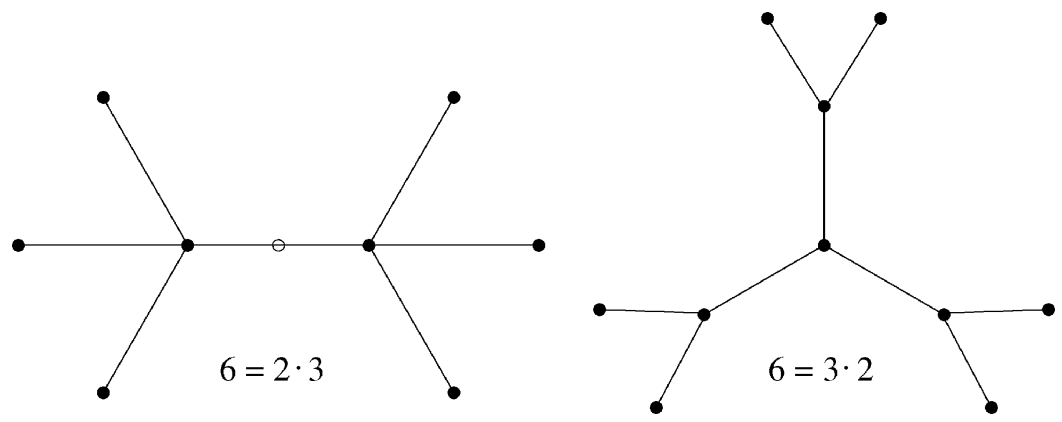

Figure 3. Two circulant trees for $k=6$.

the subsequent generations or, if the tree is bicentered, to twice the same product. Ordering differently the valencies gives rise to different trees.

We map the ordered factorisation $k=m_{1} m_{2} \ldots m_{s}$ to the tree which has a $m_{1}$-valent centre (that is a bicentre if $m_{1}=2$ ), a first generation in which each vertex has $m_{2}$ sons (and valency $m_{2}+1$ ), and so on.

See figure 3 for the trees corresponding to the two orderings of the factorisation $6=2 \cdot 3$.

The previous results describe the structure of any circulant tree and make it possible to determine its automorphism group, as follows.

Corollary 2.5 Let $\Delta$ be a circulant tree on $k$ leaves described by the ordered factorisation $\left(m_{1}, m_{2}, \ldots, m_{s}\right)\left(\right.$ with $\left.m_{1} m_{2} \ldots m_{s}=k\right)$. Then

$\operatorname{Aut}(\Delta) \simeq S_{m_{s}} 2 S_{m_{s-1}} 2 \ldots 2 S_{m_{2}} 2 S_{m_{1}}$.

Also, $p_{k}(\operatorname{Aut}(\Delta))=1$.

See [4] and [6] for details about Parker sequences for wreath products.

Using this fact, we can show that the $k$ th entry of the Parker sequence of $\operatorname{Aut}(T)$ is $a_{k}$.

It is proved in [5] that to find the $k$ th component of the Parker sequence of the automorphism group of the limit of a Fraïssé class (in our case, the class $T$ ), one may first consider the circulant unlabelled structures on $k$ points (in our case, these are the $a_{k}$ circulant trees we just found), and then sum the $k$ th entries of the Parker vectors of the automorphism groups of these circulant structures (in our case, we noted that the $k$ th component of the Parker vector of the automorphism group of a circulant tree is 1). Therefore we have the following result.

Corollary 2.6 For the class $T$ of trees, one has $p_{k}(\operatorname{Aut}(T))=a_{k}$.

We describe now a labelling of a circulant tree $\Delta$ coming from the ordered factorisation $k=m_{1} m_{2} \ldots m_{s}$, with labels from the set $\{0, \ldots, k-1\}$ such that $\Delta$ admits the cyclic automorphism $\left(\begin{array}{lllll}0 & 1 & \ldots & k-1\end{array}\right)$. 


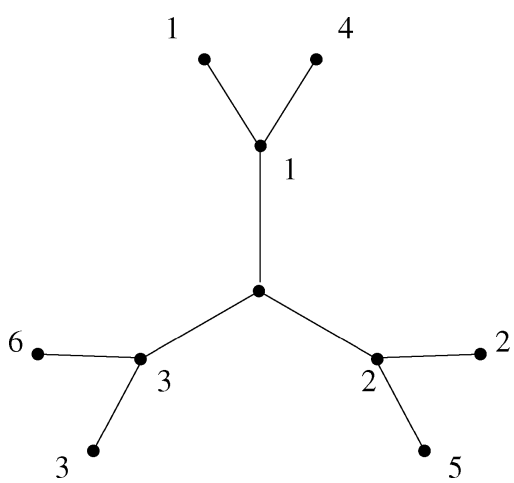

Figure 4. The labelling of a tree

If the tree is a star, i.e. $s=1$, there is a unique possible "circulant" labelling (its automorphism group being $S_{k}$ ).

Inductively, let us suppose we have described the labelling for trees with up to $n-1$ "generations" $(s \leq n-1)$. Given a tree $\Delta$ with $n$ "generations", provide $\hat{\Delta}$, which has $k / m_{s}$ leaves, with a circulant labelling with labels in $\left\{0,1, \ldots,\left(k / m_{s}\right)-1\right\}$. Now, the labelling for $\Delta$ is given as follows: the children of $i$ (leaf of $\hat{\Delta}$ ) will be named $i, i+k / m_{s}, i+2 k / m_{s}$, $\ldots, i+\left(m_{s}-1\right) k / m_{s}$ (everything modulo $\left.k\right)$. By contruction, the cycle $(01 \ldots k-1)$ is an automorphism of $\Delta$.

The labelling we just described is the only admissible one: from [5], we know that if $\Delta$ is a circulant unlabelled structure, then $p_{k}(\operatorname{Aut}(\Delta))$ gives the number of inequivalent labellings of $\Delta$ with labels from the set $\{0, \ldots, k-1\}$ such that the cycle $(0 \ldots k-1)$ is a automorphism of $\Delta$.

For boron trees, and in general for the class $T_{n}$ (trees with valency at most $n$ ), one must modify a bit the previous argument, limiting the number of trees to consider to those whose central vertex has valency lesser or equal than $n$, and all the other vertices have less than $n$ children.

For $n \geq 3$, we define a sequence $\left(a_{k}^{n}\right)$ as follows: $a_{1}^{n}=1$; for any natural $k, a_{k}^{n}=$ $\sum_{d \mid k, \frac{k}{n}<d<k} a_{d}^{n}$ (so, in particular, if $p$ is a prime, $a_{p}^{n}=1$ if $p<n$ and 0 otherwise). This is just the number of ordered factorisations of $k$ into parts strictly smaller than $n$. Note that we get again $\left(a_{k}\right)$ as "pointwise" limit of $\left(a_{k}^{n}\right)$ as $n$ tends to infinity.

Corollary 2.7 The Parker sequence of $\operatorname{Aut}\left(T_{n}\right)$ is as follows:

$$
p_{k}\left(\operatorname{Aut}\left(T_{n}\right)\right)= \begin{cases}a_{k}^{n} & \text { if } n \mid k \\ a_{k / n}^{n}+a_{k}^{n} & \text { if } n \mid k\end{cases}
$$

Proof: We are calculating the $k$-th component of the Parker sequence of $\operatorname{Aut}\left(T_{n}\right), n$ maximal valency. If $n$ does not divide $k$, the central vertex (if any) cannot have valency equal 
to $n$, and therefore the $k$-th entry of the Parker sequence is $a_{k}^{n}$. On the other hand, if $n \mid k$, in addition to the contribution given by $a_{k}^{n}$, we must consider the case in which there is a central vertex of valency equal to $n$; this case contributes $a_{k / n}^{n}$, because we must still partition the remaining $k / n$ leaves appearing in each branch.

In particular, for $T_{3}$, the class of boron trees, one has $p_{k}\left(\operatorname{Aut}\left(T_{3}\right)\right)=1$ if $k$ is of the form $3^{a} 2^{b}$ with $a \in\{0,1\}, b \geq 0$, and 0 otherwise. For $T_{n}, p_{k}$ is 0 if, and only if, $n$ is lesser than at least one of the prime factors of $k$ or it is equal to a prime factor appearing with exponent greater than 1.

\section{3. "Treelike" objects}

Let us turn now our attention to other "treelike" objects, that is to classes related to $T$ with further or different relations (see [1]).

The class $P T$ of plane trees is defined by embedding the trees in a plane; this induces a circular order on the leaves. So we have to consider the trees previously seen with the property that this additional structure is preserved by the cyclic automorphism.

Each star on $k$ leaves contributes $\varphi(k)$ to $p_{k}(\operatorname{Aut}(P T))$. In fact, suppose the leaves are $0,1, \ldots, k-1$ and the cyclic automorphism is as usual $(01 \ldots k-1)$; if the cyclic order arising from the embedding is $c_{0}<c_{1}<c_{2}<\cdots<c_{k-1}<c_{0}$, let $\sigma$ be the cyclic permutation $\left(\begin{array}{llllll}c_{0} & c_{1} & c_{2} & \ldots & c_{k-1}\end{array}\right)$. Then $\left(\begin{array}{llll}0 & 1 & \ldots & k-1\end{array}\right)$ and the $k$-cycle $\sigma$ must commute.

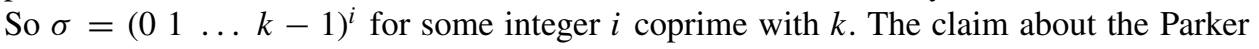
sequence follows.

Trees of other shapes cannot admit a cyclic automorphism preserving the additional circular order. In fact, if $\Delta$ is such a tree labelled as described above, in the cyclic permutation $\sigma$ describing the embedding in the plane the leaves with a common parent must appear consecutively: for instance 0 and $l k / m_{s}$, for some $l=1,2, \ldots, m_{s}-1$. So $\sigma=\left(\ldots 0 l k / m_{s} \ldots\right)$ and this $k$-cycle cannot be a power of $\left(\begin{array}{lll}0 & 1 \ldots k-1\end{array}\right)$ as required, since $l k / m_{s}$ is not coprime with $k$.

Therefore, there is no contribution from trees that are not stars, and the Parker sequence for $P T$ is the Euler function $\varphi(k)$.

For plane boron trees $\left(P T_{3}\right)$ the Parker sequence is $(1,1,2,0,0,0, \ldots)$, since a star with more than three leaves is not a boron tree.

One can define " $P T_{n}$ " as the class of plane trees with valency lesser or equal than $n$. It can be shown (see [1], p. 166) that it is not a Fraïssé class for $n>3$. One can nevertheless calculate for this class a (non-)Parker sequence; it is $\varphi(k)$ truncated to the $n$-th term.

If we consider as automorphisms the order-reversing ones as well as the order-preserving ones, we obtain the class $P^{*} T$. The $k$-leaf star contributes $\varphi(k) / 2$ to the Parker sequence, and the rest gives no contribution (in terms of groups, this mirrors what happens passing from a cyclic group to the corresponding dihedral group). For $P^{*} T_{n}$, the sequence clearly becomes $\varphi(k) / 2$ truncated to the $n$-th term.

Consider now the structures obtained by each of the above $(T, P T, \ldots)$ by distinguishing a point $\infty$ called root. In other words, we add a unary relation holding for exactly one vertex or, better, ignore the root and consider the new structure with a ternary relation $a \mid b c$ holding 


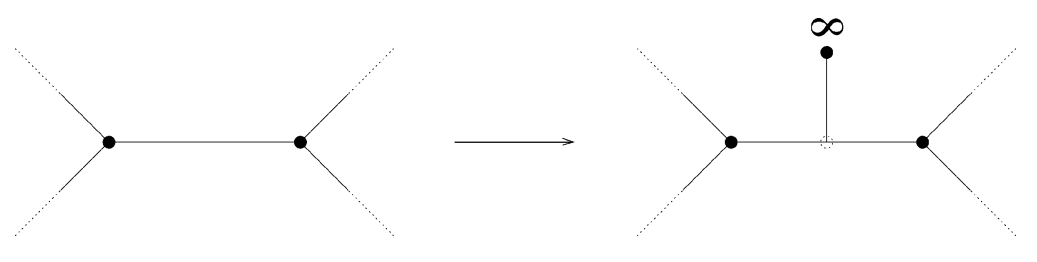

Figure 5. Adding a root.

if and only if $\infty a \mid b c$ (the knowledge of this ternary relation allows us to recover the original quaternary one). We obtain the classes $\partial T, \partial P T$, and so on.

There is a bijection between circulant trees on $k$ leaves and circulant rooted trees on $k$ leaves (that is, admitting the automorphism $(\infty)\left(\begin{array}{llll}0 & 1 & 2 \ldots k-1\end{array}\right)$, if the root is $\infty$ and the other leaves $0,1, \ldots, k-1)$. In fact, the root can be adjacent only to the centre, creating a vertex (see figure 5) if the tree is bicentered.

So the Parker sequence of $\partial T$ is the same as that of $T: p_{k}(\operatorname{Aut}(\partial T))=p_{k}(\operatorname{Aut}(T))$. We observe that $\operatorname{Aut}(\partial T)$ is isomorphic to the point stabiliser of $\operatorname{Aut}(T)$. A different kind of connection between these two groups is typical of L-sequences: $F_{k}\left(G_{\alpha}\right)=F_{k+1}(G)$.

Some care is needed when considering $\partial T_{n}$. The Parker sequence is

$$
p_{k}\left(\operatorname{Aut}\left(\partial T_{n}\right)\right)=a_{k}^{n}
$$

this can be seen by considering a (non-rooted) circulant $k$-leaf tree $\Delta$ with a central vertex of maximum valency $n$ : we cannot add to the central vertex of $\Delta$ a root (which would be its $(n+1)$ th neighbour).

For the class $\partial T_{3}(2)$ (see [1], p. 166) of the so-called Covington structures (originally introduced in [3]), the only non-zero entries of the Parker sequence are for $k=2^{i}$ (as for $\partial T_{3}$ ). For each rooted binary tree we get $2^{i}$ different circulant structures, corresponding to choosing a "colour" for the neighbour of the root, a colour for the 2 vertices at distance 2 , one for the 4 at distance 4 , and so on. So, we get as Parker sequence $p_{2^{i}}=2^{i}$, and 0 otherwise.

Let us now turn our attention to rooted plane trees. If a point in a plane tree is fixed, the circular order on the leaves is transformed in a linear order. So, $p(\operatorname{Aut}(\partial P T))=$ $(1,0,0,0, \ldots)$, because a $k$-cycle (for $k>1)\left(a_{1} a_{2} \ldots a_{k}\right)$ would map the ordered $(k+1)$ tuple $\left(\infty, a_{1}, a_{2}, \ldots, a_{k}\right)$ to $\left(\infty, a_{2}, a_{3}, \ldots, a_{k}, a_{1}\right)$. Similarly, one gets as Parker sequence for $\partial P^{*} T(1,1,0,0,0, \ldots)$.

Furthermore, $p\left(\operatorname{Aut}\left(\partial P T_{3}\right)\right)=(1,0,0,0, \ldots)$ and $p\left(\operatorname{Aut}\left(\partial P^{*} T_{3}\right)\right)=(1,1,0,0,0$, ...).

\section{Concluding remarks}

In this section we present some informal remarks, which might deserve further consideration. 
Most groups act on a class of circulant structures more trivially than on, say, sets or $n$-tuples: this depends both on the fact that there are less objects on the set on which we are acting and on the fact that the circulant structures are by definition quite regular.

The Parker sequences of oligomorphic groups display nevertheless several kinds of behaviour. Empirically, the sequences seem to fall into three main families (and some sporadic examples, as the sequence for $K_{3}$-free graphs; see [6]).

1. Trivial sequences, in the sense that $\sum_{k} p_{k}<\operatorname{deg} G$, that is, there is only a finite number of non-zero terms in the sequence;

2. Sequences whose $k$-th term is related to the factorisation of $k$;

3. Sequences with at least exponential growth.

Arguments related to the factorisation of $k$ often occur, leading mostly to Parker sequences whose terms are either factorisations of $k$ with suitable properties or $\varphi$-related functions.

The first situation, typical for instance of trees and variations thereof, arises when each isomorphism type of circular structures provides exactly one structure (= contributes exactly 1 to $\left.p_{k}\right)$. In terms of cycles, this is related to what happens for the symmetric groups, characterised in the finite case by having an all-1 Parker vector. Another example in the same vein is given by $S^{2}$ in its product action (see [6]).

The second situation arises when on any given $k$-structure something acts as the cyclic group, that is, if some circular order is the defining structure. The classical example is given by the group $C$ (preserving a circular order on a countable set; see [2] and [5]); another example is $\operatorname{Aut}(P T)$.

The sequences growing exponentially, of the form $a^{f(k)}$, arise from counting maps from a set of size $f(k)$ (symbols for graphs etc.) to a set of size $a$ (often $\{0,1\}$ or a set of orientations, colours...). An example in this paper is provided by Covington structures; other more standard examples come from graphs and hypergraphs and, more generally, from $n$-ary relations.

\section{References}

1. P.J. Cameron, "Some treelike objects," Quart. J. Math. Oxford 38(2) (1987), 155-183.

2. P.J. Cameron, Oligomorphic Permutation Groups, LMS Lecture Notes 152, Cambridge Univ. Press, Cambridge, 1990.

3. J. Covington, "A universal structure for $N$-free graphs," Proc. London Math. Soc. 58(3) (1989), 1-16.

4. D.A. Gewurz, "Parker vectors and cycle indices of permutation groups," Quaderni Elettronici del Seminario di Geometria Combinatoria 4E (2002) [http://www.mat.uniroma1.it/ combinat/ quaderni].

5. D.A. Gewurz and F. Merola, "Parker vectors for infinite groups," Europ. J. Combin. 22 (2001), 1065-1073.

6. D.A. Gewurz and F. Merola, "Sequences realised as Parker vectors of oligomorphic permutation groups," Journal of Integer Sequences 6 (2003), Article 03.1.6 (electronic) [http://www . math.uwaterloo.ca/JIS/].

7. D.E. Knuth, Axioms and Hulls, Lecture Notes in Computer Science 606, Springer-Verlag, Heidelberg, 1992.

8. H.D. Macpherson, "Orbits of infinite permutation groups," Proc. London Math. Soc. 51(3) (1985), $246-284$.

9. F. Merola, "Orbits on $n$-tuples for infinite permutation groups," European J. Combin. 22 (2001), 225-241.

10. N.J.A. Sloane (Ed)., The On-Line Encyclopedia of Integer Sequences, published electronically at http://www.research.att.com/ njas/sequences/. 